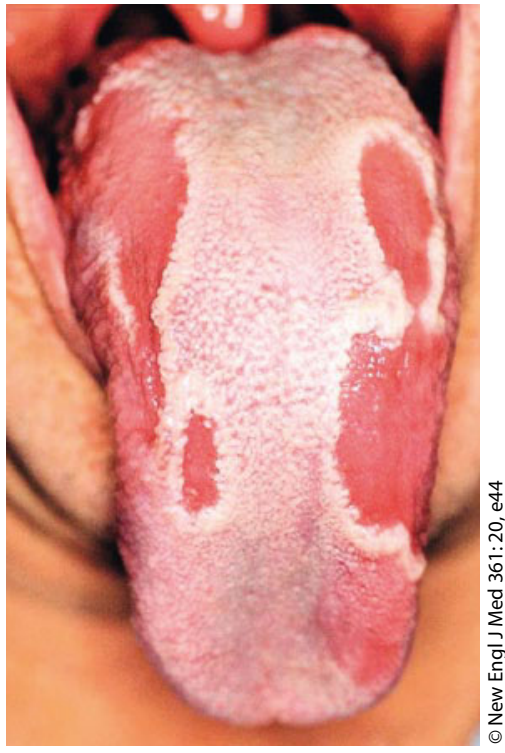

Eine benigne migratorische Glossitis.

\section{Ist das gefährlich?}

- Ein 61-jähriger Mann wurde wegen schmerzloser weißlicher Beläge auf der Zunge überwiesen, die ihm vor einem Monat aufgefallen waren. Er war bereits topisch und systemisch mit Antimykotika wegen des Verdachts auf eine orale Kandidiasis behandelt worden, die Läsionen blieben aber unverändert.

Der Patient berichtete, er habe vor einem Jahr bereits ähnliche Erscheinungen gehabt, die sich aber spontan zurückgebildet hätten. Bei der Untersuchung erkannte man multiple rötliche Areale der Zunge mit ringförmigem, scharf begrenztem Rand. Die Diagnose einer Lingua geographica wurde gestellt.

Die Lingua geographica oder benigne migratorische Glossitis ist eine gutartige entzündliche Veränderung der Zunge, die bei etwa 2\% der Weltbevölkerung auftritt. Die typische Manifestation ist eine landkartenartige Verteilung eines Erythems, das durch eine Atrophie der filiformen Zungenpapillen hervorgerufen wird, die von einem weißen hyperkeratotischen Saum begrenzt werden. Die Läsionen bilden sich üblicherweise spontan und folgenlos zurück, können aber an anderen Stellen erneut auftreten.

Ein typischer Fall für den Blickdiagnostiker, der viele Odysseen dieser Patienten vermeiden kann.

H. S. FÜESSL =

- E. Masferrer und A. Jucgla

Geographic tongue. New Engl J Med 361: 20, e44

\title{
Antipsychotische Therapie erhöht das Thromboembolierisiko
}

Es gab bisher nur eine Reihe von kleineren Beobachtungsstudien, die unterstellen, dass Neuroleptika zu einem erhöhten Risiko von venösen Thromboembolien führen.

— In einer englischen Fall-Kontroll-Studie wurden Patienten erfasst, die zwischen Januar 1996 und Juli 2007 eine venöse Thromboembolie erlitten hatten. Jeder dieser Patienten wurde dann mit vier gematchten Kontrollen verglichen. Der primäre Outcome war die Odds Ratio für venöse Thromboembolien bei Patienten, die antipsychotische Medikamente einnahmen.

Die Studie stützte sich auf eine Population von über sieben Millionen Menschen. Darunter fanden sich 25532 Patienten mit venösen Thromboembolien. Diese Fälle wurden 89491 gematchten Kontrollen gegenüber gestellt.

Patienten, die in den vergangenen 24 Monaten ein Neuroleptikum verschrie- ben bekommen hatten, hatten ein 32\% erhöhtes Risiko venöser Thromboembolien verglichen mit Patienten, die diese Medikamente nicht eingenommen hatten.

Ein signifikant erhöhtes Risiko fand sich bei derzeitiger Einnahme von Neuroleptika bei Patienten, die Neuroleptika zum ersten Mal einnahmen, bei Patienten, die Neuroleptika längere Zeit einnahmen und bei Patienten, die Neuroleptika in einem Zeitraum von 4-12 Monaten zuvor eingenommen hatten. Patienten, bei denen die Einnahme der Neuroleptika länger als zwölf Monate zurücklag, hatten kein erhöhtes Risiko mehr.

Patienten mit konventionellen Neuroleptika hatten ein 28\% erhöhtes Risiko, Patienten mit atypischen Neuroleptika ein 73\% erhöhtes Risiko. Hochpotente Neuroleptika hatten interessanterweise ein geringeres Risiko als niederpotente Neuroleptika. Innerhalb der Grup- pe der Neuroleptika hatten Haloperidol und Quetiapin das höchste Risiko.

\section{Kommentar}

Diese sehr große Fall-Kontroll-Studie belegt zum ersten Mal relativ eindeutig, dass ein Zusammenhang zwischen der Einnahme von Neuroleptika und venösen Thromboembolien besteht. Für den klinischen Alltag ist es besonders wichtig, dass das Risiko von venösen Thromboembolien mit dem Alter ansteigt und die hier gefundenen Ergebnisse insbesondere Menschen im Alter über 75 Jahren betrifft. Leider wurde nicht untersucht, ob eine Anamnese von venösen Thrombosen zu einem erhöhten Risiko unter Einnahme von Neuroleptika prädisponiert. Dies hätte die Aufklärung von Patienten über das potenzielle Risiko deutlich erleichtert.

H.-C. DIENER =

- C. Parker , C. Coupland , J. Hippisley-Cox Antipsychotic drugs and risk of venous thromboembolism: nested case-control study. BMJ 341 (2010) c4245 